\title{
Effects of Altitude on Thermal Flying-Height Control Actuation
}

\author{
Jinglin Zheng • David B. Bogy • \\ Shuyu Zhang $\cdot$ Wentao Yan
}

Received: 4 September 2009/ Accepted: 10 July 2010/Published online: 22 July 2010

(C) The Author(s) 2010. This article is published with open access at Springerlink.com

\begin{abstract}
Thermal flying-height control (TFC) is now a key technology used in hard-disk drives (HDD) as an effective way to push the magnetic spacing to sub-5 $\mathrm{nm}$. Precise control of the TFC sliders' actuated flying-height $(\mathrm{FH})$ is a major consideration in improving the read/write capability as well as reducing the reliability problem. In this paper, we investigate the response of TFC sliders to altitude changes with a focus on the variation of actuated FH. Numerical and experimental results both indicate an increase in the actuated FH at higher altitudes. Simulations show that the increased TFC protrusion and less air bearing pushback on sliders at higher altitudes contribute to this increase. This study is of practical importance for improving heater and air bearing surface design to reduce the TFC sliders' sensitivities to altitude changes.
\end{abstract}

Keywords Thermal flying-height control · Altitude · Protrusion · Pushback

\section{Introduction}

The ever-increasing demand for higher areal density in hard-disk drives has pushed the magnetic spacing to sub$5 \mathrm{~nm}$. Among the technologies enabling sliders to fly lower, the thermal flying-height control (TFC) technology

J. Zheng $(\bowtie) \cdot$ D. B. Bogy

Computer Mechanics Laboratory, Department of Mechanical

Engineering, University of California at Berkeley, Berkeley,

CA 94720, USA

e-mail: zhengjinglin0420@gmail.com

S. Zhang · W. Yan

Magnetic Head Operations, Western Digital Corporation,

44200 Osgood Road, Fremont, CA 94539, USA has become the most popular one [1, 2]. As illustrated in Fig. 1, the slider is locally heated by an imbedded heater near the read-write transducers so that a heater-induced protrusion is formed on the air bearing surface (ABS). The original flying-height $(\mathrm{FH})$ at the read-write transducers can be reduced as much as several nanometers under such an actuation.

However, with this reduced $\mathrm{FH}$, the read-write transducers can potentially be degraded or even damaged due to contact with the disk induced by the FH variations related to several factors, such as the external environmental conditions, dynamic disturbances, manufacturing tolerance, etc. Efforts have been made to address the issues of these effects [3-7]. Yet none of these studies covers the case of the altitude sensitivity of TFC sliders.

In this paper, we focus on the altitude effects on the TFC actuation. We numerically calculate the actuated FH at different altitudes with a model described in Sect. 2, and validate this model with drive level testing data. We investigate the mechanisms causing the variations in actuated FH's using numerical simulations and found that the increased protrusion and weakened pushback from the air bearing are the contributing factors. The results presented here are of practical importance for improving the TFC sliders' performance under environmental changes.

\section{Numerical Approach}

\subsection{Model and Solution}

The numerical approach used in this analysis involves two steps: solving the TFC protrusion at a given actuation power, and solving the flying attitude of the protruded 


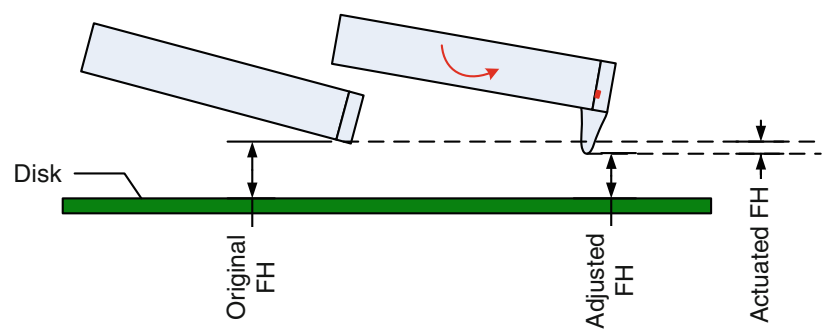

Fig. 1 Illustration diagram of thermal flying-height control sliders. The flying-height at the read-write transducers is adjusted by the TFC protrusion at the trailing edge. The original $\mathrm{FH}$ is defined as the slider's flying-height with the heater turned off. The adjusted FH is the slider's flying-height with the heater turned on. The actuated FH is defined as the difference between the original $\mathrm{FH}$ and the adjusted $\mathrm{FH}$

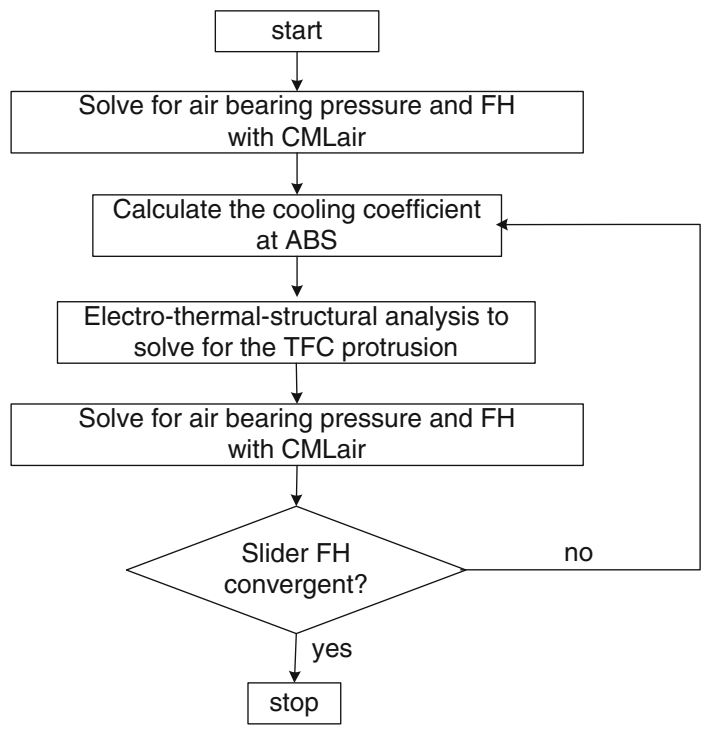

Fig. 2 Flowchart for the iterative approach of TFC simulations

slider. One tricky problem in TFC simulations is the following coupling relationship: on the one hand, the protrusion geometry is strongly dependent on the air bearing pressure as the air bearing pressure affects the heat dissipation at the air bearing surface; on the other hand, the air bearing pressure, in turn, is determined by the air bearing surface design, thus it is affected by the protrusion geometry. Therefore, an iterative procedure is necessary for an accurate numerical solution [8]. Figure 2 shows a flowchart of the iteration method used in this study.

Specifically, we first use CMLair, a finite volume solver [9], to solve the generalized Reynolds equation and obtain the air bearing pressure and flying-height. Then the heat transfer model developed in [10] is used to determine the heat dissipation at the slider's surface. According to [10], the air bearing cooling is the dominating factor in the heat dissipation at a flying slider's surface. Therefore, it plays an important role in determining the TFC protrusion amount. The cooling coefficient $h$ due to the air bearing is: $h(x, y)=\frac{k}{d(x, y)+2 b \lambda(x, y)}$

where, $k$ is the thermal conductivity of air and is equal to $0.0261 \mathrm{~W} /(\mathrm{m} \mathrm{K})$ at $298 \mathrm{~K}, d$ is the slider's flying-height at a local point, which is obtained from CMLair, $\lambda$ is the mean free path of air molecules in the air gap, and is equal to $68.6 \mathrm{~nm}$ at $298 \mathrm{~K}$ and $1 \mathrm{~atm}$. Note that the flying-height $d$ and the mean free path $\lambda$ in Eq. 1 vary with the location $(x, y) . \lambda$ is inversely dependent on the local air bearing pressure, as shown by the US standard atmosphere model [11]:

$\lambda=\frac{2^{1 / 2} \cdot R^{*} \cdot T}{2 \pi \cdot N_{\mathrm{A}} \cdot \sigma^{2} \cdot P(x, y)}$

where, $R^{*}=8.31432 \mathrm{~J} /(\mathrm{mol} \mathrm{K})$ is the gas constant, $T$ is the ambient temperature and is equal to $298 \mathrm{~K}, N_{\mathrm{A}}=$ $6.02213 \times 10^{23}$ is the Avogadro constant, $\sigma=3.65 \times$ $10^{-10} \mathrm{~m}$ is the effective collision diameter of the air molecules, $P(x, y)$ is the pressure at a local point $(x, y)$ in the air bearing.

The coefficient $b$ in Eq. 1 is defined by:

$b=\frac{2\left(2-\sigma_{\mathrm{T}}\right) \gamma}{\sigma_{\mathrm{T}}(\gamma+1) \operatorname{Pr}}$

where, $\sigma_{\mathrm{T}}$, set equal to 0.9 , is the thermal accommodation coefficient, $\gamma=1.4$ is the specific heat ratio for air, and $\operatorname{Pr}=0.707$ is the Prandtl number for air at $298 \mathrm{~K}$.

Next we solve for the TFC protrusion by conducting an electro-thermal-structural analysis in ANSYS, a commercial finite element software. The actuation power is applied by putting voltage loads on the pads of the TFC heater. The air bearing cooling coefficients calculated from Eq. 1 are applied as thermal boundary conditions in the finite element model built in ANSYS. The protruded air bearing surface obtained from this analysis is used in the next round of pressure and FH calculation in CMLair. Such a process is continued until the variation in flying-height is less than $0.05 \mathrm{~nm}$. Once this criterion is reached, the slider's flying-height at the actuated state is determined as well as the actuated FH. In this study, we define the actuated $\mathrm{FH}$ as the $\mathrm{FH}$ change at the read transducer between the actuated state and the non-actuated state.

\subsection{Experimental Verification of the Model}

To verify the model in Sect. 2.1, we ran a series of experiments and compared the simulation results with the test data. These experiments utilized the measurement of the read-back signal of the magnetic head in commercial disk drives operating in an altitude chamber. The chamber pressure can be set to different values (altitude related). The read-back signal was converted to the $\mathrm{FH}$ changes at 
the read transducer by applying the Wallace spacing loss theory [12]. Specifically, the magnetic spacing variation due to the thermal actuation can be calculated by [13]:

$\bar{d}_{1}-\bar{d}_{2}=-\frac{r m}{f} \ln \frac{E\left(\bar{d}_{1}\right)}{E\left(\bar{d}_{2}\right)}$

where $\bar{d}$ is the magnetic spacing at a specific actuation power, $m$ is the disk rotating speed, $r$ is the track radius, $f$ is the recording frequency, $E$ is the amplitude of the readback voltage and it varies with the magnetic spacing $\bar{d}$. The FH change at the read transducer is equal to the magnetic spacing change, so we can calculate the FH change at the read transducer between any two actuation powers by applying Eq. 4. Thus, the actuated $\mathrm{FH}$ is determined experimentally as:

Actuated $\mathrm{FH}=-\frac{r m}{f} \ln \frac{E_{\text {actuated }}}{E_{\text {non-actuated }}}$

although the absolute value of the read transducer $\mathrm{FH}$ in the non-actuated state cannot be measured. The actuated FH at a specific actuation power is measured repeatedly and the average value is taken to compare with the simulation result. The standard deviation $\sigma$ of the measured actuated $\mathrm{FH}$ varies with the actuation power. However, $\sigma$ is within $5 \%$ of the average actuated FH.

Figure 3 shows a comparison of the actuated FH from simulations and experiments at two different altitudes: sea level and $3 \mathrm{~km}$, for a femto-sized slider (length $\times$ width $\times$ height $=0.85 \times 0.70 \times 0.23 \mathrm{~mm})$. The results show that the actuated $\mathrm{FH}$ is greater at an altitude of $3 \mathrm{~km}$ than at sea level. Simulations and experiments are seen to be in excellent agreement for the investigated design.

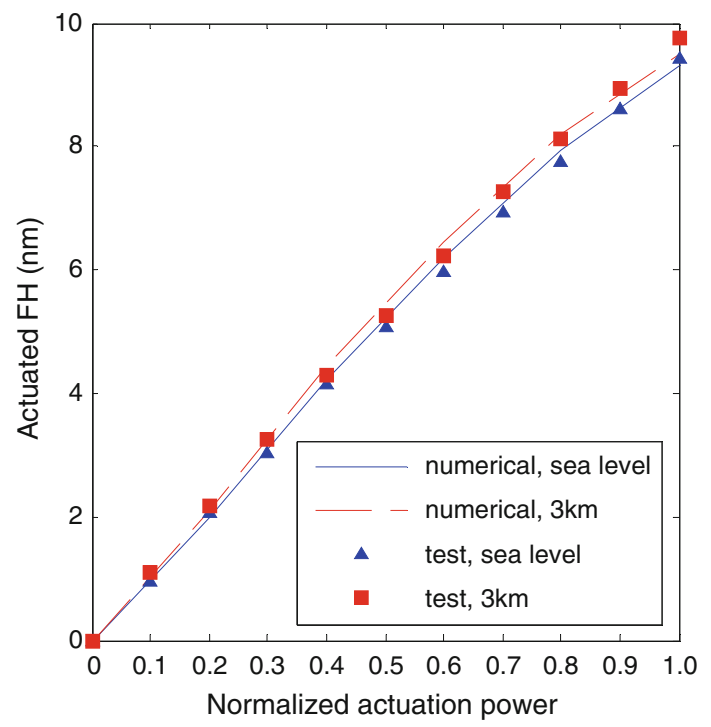

Fig. 3 Actuated FH at the read transducer vs. normalized actuation power. Numerical and test results at the sea level and $3 \mathrm{~km}$ are presented

\section{Results and Analysis}

One important feature of the thermal flying-height control technology is that the actuated FH is usually not the same as the TFC protrusion amount. The air bearing pressure at the protruded area usually goes up significantly and pushes the slider away from the disk. To characterize this phenomenon, a pushback factor $\alpha$ is introduced in and its relationship with the actuated $\mathrm{FH}$ is defined by Eq. 6:

Actuated FH $=$ TFC protrusion $\cdot(1-\alpha)$

All parameters in Eq. 6 are evaluated at the read transducer location. As altitude changes cause variations in both the TFC protrusion and the pushback factor, we investigate these two aspects individually in the following paragraphs.

\subsection{Effects of Altitude on the TFC Protrusion}

Figure 4 shows the simulated TFC protrusion along a line parallel to the $y$-direction (slider width direction) and passing through the read transducer $\left(x=x_{\text {transducer }}\right)$. The curves are obtained with a normalized actuation power of 1. Results of sea level and $4 \mathrm{~km}$ altitude are shown. The location of the read transducer is indicated in the figure. The protrusion is larger at the higher altitude. Since the heater design and the actuation power are the same, this implies that the air bearing cooling is weaker at the higher altitude.

In Fig. 5, we show the cooling coefficient $h$ along the line $x=x_{\text {transducer }}$ for these two altitudes. An obvious difference can be observed at the transducer location, showing the cooling coefficient is reduced at the higher altitude.

According to Eq. 1, the air bearing cooling coefficient $h$ is determined by the local FH $d(x, y)$ and mean free path $\lambda(x, y)$. Both $d$ and $\lambda$ vary with altitude. On the one hand,

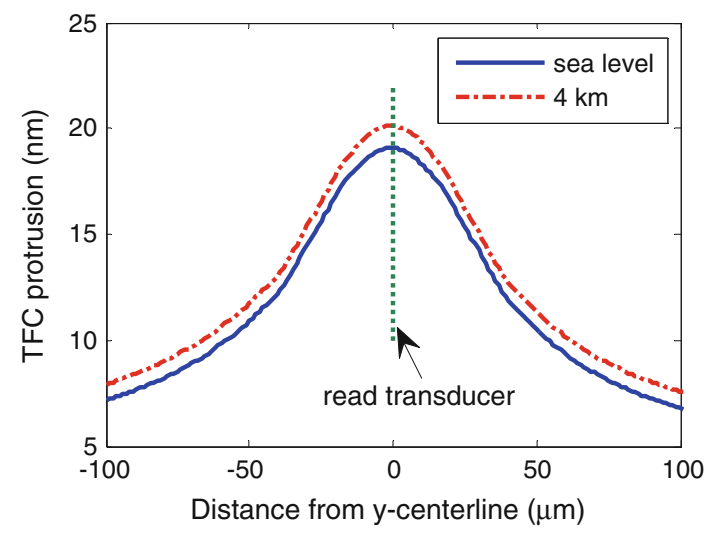

Fig. 4 The TFC protrusion along the line $x=x_{\text {transducer }}$ with a normalized actuation power of 1 at two altitudes: sea level and $4 \mathrm{~km}$. The location of the read transducer is indicated in the figure 


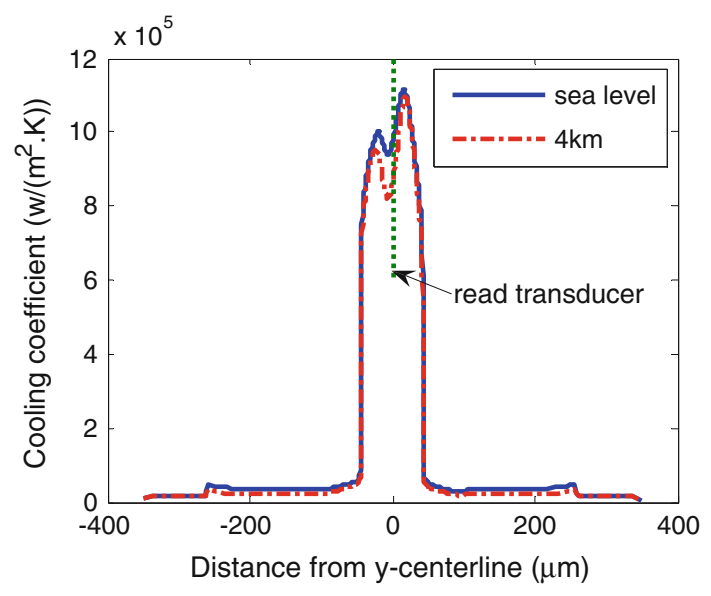

Fig. 5 The air bearing cooling coefficient $h$ along the line $x=$ $x_{\text {transducer }}$ with a normalized actuation power of 1 at two altitudes: sea level and $4 \mathrm{~km}$. The location of the read transducer is indicated in the figure

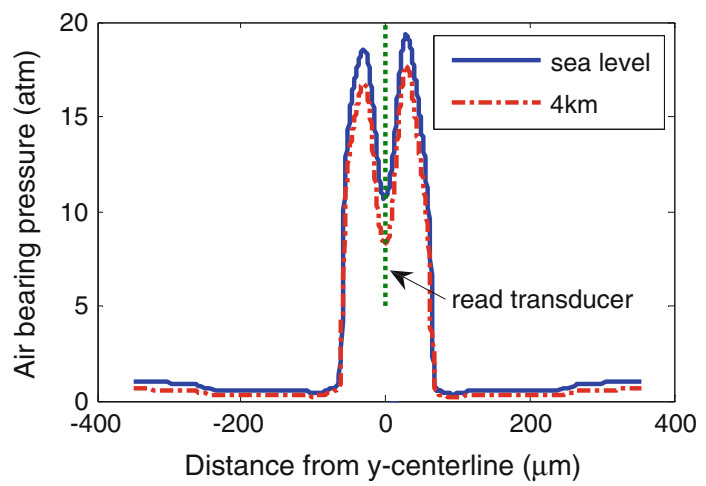

Fig. 6 The air bearing pressure along the line $x=x_{\text {transducer }}$ with a normalized actuation power of 1 at two altitudes: sea level and $4 \mathrm{~km}$. The location of the read transducer is indicated in the figure

the local $d$ at the transducer area is smaller (usually, but not always) at higher altitudes, and this enhances the heat dissipation, as predicted by Eq. 1. On the other hand, the pressure at the transducer area is smaller at the higher altitude, as shown in Fig. 6, which increases the local mean free path $\lambda$, as shown in Eq. 2, and this reduces the local cooling coefficient $h(x, y)$, as predicted by Eq. 1. Although altitude-induced changes in $d$ and $\lambda$ at the transducer area have offsetting effects on the cooling coefficient $h$, it is apparent that the effect of increased $\lambda$ dominates in the investigated case. Physically, this means that the heat dissipation at the transducer area is reduced mainly because fewer molecules exist in the air gap to provide energy exchanges.

\subsection{Effects of Altitude on the Pushback Factor $\alpha$}

As pushback is a response of the air pressure to the protruded air bearing surface, it is related to the altitude, as

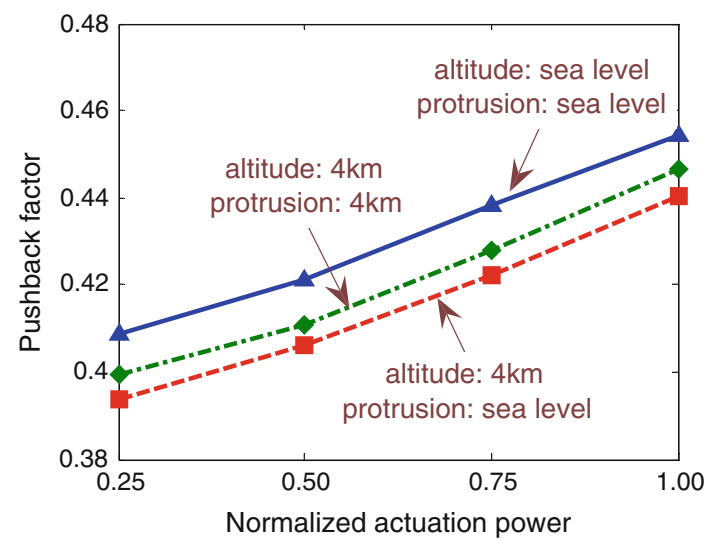

Fig. 7 The pushback factor vs. normalized actuation power. Three cases are investigated: (i) the slider flying at sea level with the protrusions obtained at sea level; (ii) the slider flying at $4 \mathrm{~km}$ with the protrusions obtained at $4 \mathrm{~km}$; (iii) the slider flying at $4 \mathrm{~km}$ with the protrusions obtained at sea level

well as the protrusion. To separate the effect of the protrusion, we apply the sea level protrusion profile on the slider flying at sea level and $4 \mathrm{~km}$, respectively. As shown in Fig. 7, the pushback factor goes up with increasing actuation powers, and experiences higher values at sea level than at $4 \mathrm{~km}$ given the same protrusion. This result implies that the pressure change due to the TFC protrusion is smaller at higher altitude than at sea level. Thus it requires less pushback to regain the equilibrium at altitude [5]. To verify this, we fix the slider at $12 \mathrm{~nm} \mathrm{FH}$ at its trailing edge center, with $90 \mu \mathrm{rad}$ pitch angle and 0 roll angle, then modify the air bearing surface with sea level protrusions at increasing actuation powers. As shown in Fig. 8, the rate of change in the air bearing force with increasing actuation power is higher at sea level, showing a stronger stiffening response of the air bearing. Accordingly, to balance the suspension load, the air bearing has to push the slider up farther so that the increase in the air bearing force can be compensated.

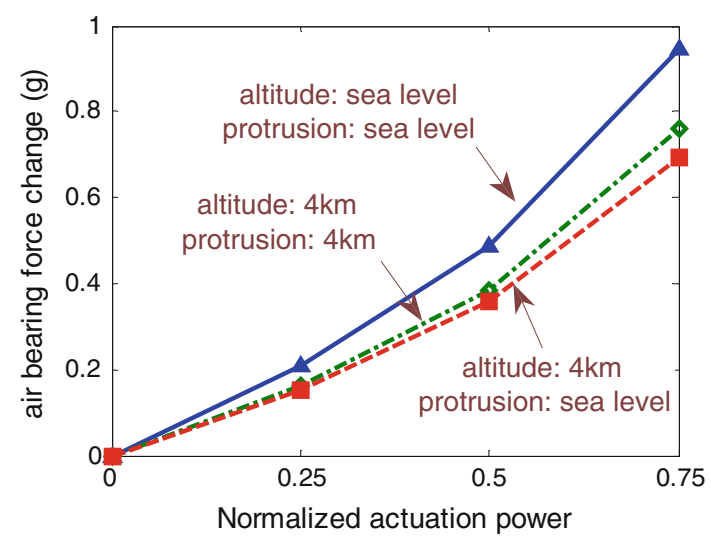

Fig. 8 The air bearing force change vs. normalized actuation power. The same three cases are investigated as in Fig. 7 


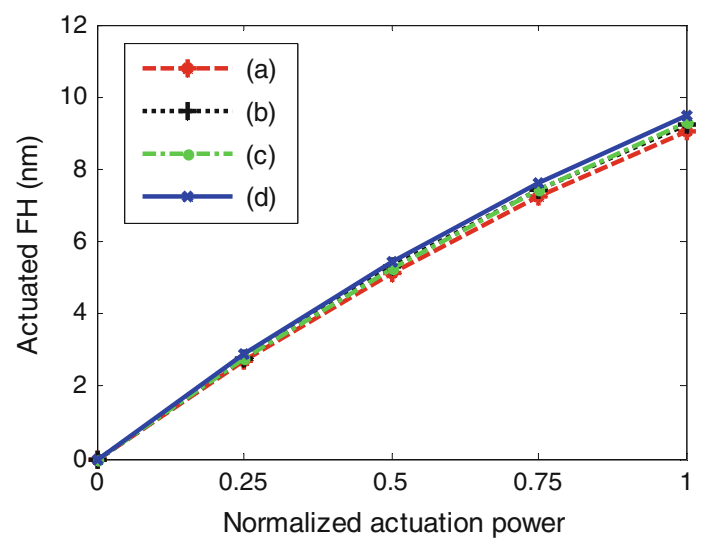

Fig. 9 Actuated FH vs. normalized actuation power for: $(a)$ the slider flying at sea level with sea level protrusions; $(b)$ the slider flying at sea level with $4 \mathrm{~km}$ protrusions; (c) the slider flying at $4 \mathrm{~km}$ with sea level protrusions; $(d)$ the slider flying at $4 \mathrm{~km}$ with $4 \mathrm{~km}$ protrusions

On the other hand, if the $4 \mathrm{~km}$ protrusion is used for the slider flying at $4 \mathrm{~km}$ altitude in Fig. 7, the resultant pushback factor is higher compared with the sea level protrusion $/ 4 \mathrm{~km}$ altitude case. This is reasonable as we have shown in Sect. 3.1 that the $4 \mathrm{~km}$ protrusion is larger, so stronger pushback is expected. In addition, the pushback factor is still lower compared with the sea level protrusion/ sea level altitude case. This indicates that the altitudeinduced change in the air bearing pressure is the dominating factor in determining the pushback factor. Figure 8 also shows the fixed-altitude simulation results for the $4 \mathrm{~km}$ protrusion $/ 4 \mathrm{~km}$ altitude case. The result is consistent with Fig. 7 as the rate of change in the air bearing force for the $4 \mathrm{~km}$ protrusion $/ 4 \mathrm{~km}$ altitude case is higher than the sea level protrusion $/ 4 \mathrm{~km}$ altitude case, but lower than the sea level protrusion/sea level altitude case.

In the proceeding paragraphs, we analyzed the variation of the TFC protrusion and pushback factor under altitude changes. The increased protrusion and reduced pushback factor both contribute to the increased actuated $\mathrm{FH}$ at higher altitudes. To quantify these two contributions, we compare the actuated FH of the following four cases: (a) the slider flying at sea level with sea level protrusions; (b) the slider flying at sea level with $4 \mathrm{~km}$ protrusions; (c) the slider flying at $4 \mathrm{~km}$ with sea level protrusions; (d) the slider flying at $4 \mathrm{~km}$ with $4 \mathrm{~km}$ protrusions. A comparison between (a) and (b) reveals how much the variation in the TFC protrusion alone affects the actuated FH. A comparison between (a) and (c) tells us the effects of reduced pushback on the actuated FH. The difference between (a) and (d) is the combined effects of these two. In Fig. 9, the curves for cases (b) and (c) almost overlap, showing that the two factors have roughly equivalent effects for this investigated design.

\section{Conclusion}

The effect of altitude on thermal flying-height control sliders is investigated numerically and experimentally. Both simulations and experiments indicate that the actuated $\mathrm{FH}$ is larger at increased altitude. It is found through simulations that such an increase is due to the increased protrusion and less pushback at a higher altitude. The former is caused by the reduced cooling at the transducer area. The latter is associated with the reduced lift force change from the air bearing. These results are of practical importance for heater and ABS design in order to reduce the sliders' sensitivities to altitude and thus improve the HDI stabilities under ambient condition changes.

Open Access This article is distributed under the terms of the Creative Commons Attribution Noncommercial License which permits any noncommercial use, distribution, and reproduction in any medium, provided the original author(s) and source are credited.

\section{References}

1. Aoki, K., Hoshino, T., Iwase, T., Imamura, T., Aruga, K.: Thermal pole-tip protrusion analysis of magnetic heads for hard disk drives. IEEE Trans. Magn. 41, 3043-3045 (2005)

2. Aoki, K., Watanabe, T.: Nonlinearity of thermal spacing control in hard disk drives. IEEE Trans. Magn. 45, 816-821 (2009)

3. Cha, E., Chiang, C., Enguero, J., Lee, J.J.K.: Effect of temperature and altitude on flying-height. IEEE Trans. Magn. 32, 3729-3731 (1996)

4. Zhang, S., Strom, B., Lee, S.C., Tyndall, G.: Simulating the air bearing pressure and flying height in a humid environment. J. Tribol. 130, 011008 (2008)

5. Zhang, S., Lee, S.C., Kim, D., Ferber, J., Strom, B., Tyndall, G.: Air bearing surface designs in consideration of thermomechanical actuation efficiency. J. Tribol. 130, 041901 (2008)

6. Strom, B.D., Zhang, S., Lee, S.C., Khurshudov, A., Tyndall, G.W.: Effects of humid air on air-bearing flying-height. IEEE Trans. Magn. 43, 3301-3304 (2007)

7. Miyake, K., Shiramatsu, T., Kurita, M., Tanaka, H., Suk, M., Saegusa, S.: Optimized design of heaters for flying height adjustment to preserve performance and reliability. IEEE Trans. Magn. 43, 2235-2237 (2007)

8. Juang, J., Chen, D., Bogy, D.B.: Alternate air bearing slider designs for areal density of $1 \mathrm{~Tb} / \mathrm{in}^{2}$. IEEE Trans. Magn. 42, 241-246 (2006)

9. Cox, B.J., Bogy, D.B.: The CML Air Bearing Design Program (CMLAir), Version 7 User Manual (2007)

10. Chen, L., Bogy, D.B., Strom, B.: Thermal dependence of MR signal on slider flying state. IEEE Trans. Magn. 36, 2486-2489 (2000)

11. U.S. standard atmosphere, 1-20 (1976)

12. Wallace, Jr., R.J.: The reproduction of magnetically recorded signals. Bell. Syst. Tech. J. 30, 1145-1173 (1951)

13. Shi, W.K., Zhu, L.Y., Bogy, D.B.: Use of readback signal modulation to measure head/disk spacing variations in magnetic disk files. IEEE Trans. Magn. 23, 233-240 (2000) 